\title{
MÉTODOS CONTRACEPTIVOS: A PRÁTICA DOS ADOLESCENTES DAS ESCOLAS AGRÍCOLAS DA UNIVERSIDADE FEDERAL DO PIAUÍa
}

\author{
Contraceptive Methods: The Practice of Adolescents from \\ Agrarian Schools of Federal University of Piauí

\section{Métodos Anticonceptivos: La Práctica de los Adolescentes de las Escuelas Agrícolas de la Universidad Federal de Piauí}

Rita de Cássia Magalhães Mendonça ${ }^{1}$

Telma Maria Evangelista Araújo²

\begin{abstract}
RESUMO
Este estudo objetivou analisar a prática relacionada ao uso dos métodos contraceptivos entre adolescentes das escolas agrícolas da Universidade Federal do Piauí. Realizou-se estudo transversal com 652 adolescentes com idades entre 14 e 19 anos. Os resultados mostraram que, no sexo feminino, a idade média na primeira relação sexual foi de 15 anos (37,5\%), com parceiro estável (71,9\%). No sexo masculino, 76,7\% iniciaram sua atividade sexual entre 14 e 15 anos, com parceira casual (73,5\%). 0 contraceptivo mais conhecido por ambos os sexos foi o condom. Observou-se associação entre o conhecimento e uso da pílula $(p<0,05)$. Concluiu-se que a saúde sexual do adolescente precisa ser mais discutida no contexto político. Deve-se ressaltar a necessidade de a escola enfocar a saúde sexual e preventiva.
\end{abstract}

Palavras-chave: Adolescente. Anticoncepcionais. Enfermagem.

\begin{abstract}
This study aimed to analyze the practice of adolescents from the Agrarian Schools of the Federal University of Piauí, when it comes to the contraceptive methods. It is a transversal study performed with 652 adolescents aged from 14 to 19 years old. The results showed that the first intercourse happened mostly around 15 years old, in the female gender, (37.5\%), with a steady partner $(71.9 \%)$. When it comes to the male gender, $76.7 \%$ have already begun their sexual activity around 14-15 years old, with an unsteady partner (73.5\%). The most known contraceptive method by the adolescents from both genders was the condom. The association between the knowledge and the usage of pill $(p<0.05)$ was observed. We concluded that the adolescent's sexual health needs to be better discussed in the political context. We must emphasize the necessity for the school to focus on the sexual and preventive health.
\end{abstract}

Key words: Adolescence. Contraceptives. Nursing.

\section{Resumen}

El estudio objetivó analizar la práctica de los adolescentes de las Escuelas Agrícolas de la Universidad Federal de Piauí en relación al uso de los métodos anticonceptivos . Es un estudio transversal con 652 adolescentes de 14 a 19 años. Los resultados mostraron que, en las mujeres, la edad promedio en que se presentó la primera relación sexual a los 15 años $(37,5 \%)$, con un compañero estable $(71,9 \%)$. En el sexo masculino, 76,7\% comenzaron su actividad sexual a los 14 15 años, con una compañera casual $(73,5 \%)$. El método anticonceptivo más conocido por los adolescentes de ambos los sexos fue el condón. Observamos la asociación entre el conocimiento y el uso de la píldora $(p<0,05)$. Se concluyó que la salud sexual del adolescente necesita ser más ampliamente discutida en el contexto político. Cabe destacar que las escuelas deben focalizar esfuerzos en la salud sexual y preventiva. .

Palabras clave: Adolescente. Anticonceptivos. Enfermería.

'Mestre em Enfermagem, Coordenadora e Professora do Curso Técnico em Enfermagem do Colégio Agrícola da Universidade Federal do Piauí - UFPI. Brasil. E-mail: ritamagalhes01@oi.com.br, '2Doutora em Enfermagem, Professora do Departamento de Enfermagem da Universidade Federal do Piauí UFPI e da NOVAFAPI. Coordenadora do Programa de Pós-graduação Mestrado em Enfermagem da UFPI. Brasil. E-mail: telmaevangelista@gmail.com 
Métodos Contraceptivos: A prática dos adolescentes Mendonça RCM, Araújo TME

\section{INTRODUCÃO}

A adolescência é uma fase especial na vida humana que desperta interesse em diversos segmentos da sociedade, tendo em vista ser um processo de mudança anatômica, fisiológica, emocional e comportamental que interfere na formação da personalidade. Neste sentido, é uma fase considerada preocupante em relação à saúde sexual e reprodutiva em virtude da possibilidade da gravidez indesejada e precoce, além da exposição às doenças sexualmente transmissíveis (DSTs) e a AIDS. Assim, o tema induz discussões nas políticas de educação sexual e reprodutiva do adolescente.

0 termo adolescência representa o período de crescimento e desenvolvimento biológico, psicológico e social o qual os seres humanos experimentam na vida, de uma forma dinâmica e em curto período de tempo, em que as mudanças no corpo físico assumem um caráter complexo. Para alguns adolescentes é relativamente fácil absorver essas mudanças, enquanto a maioria necessita de um tempo para adaptação, visto que estas modificações possuem uma relação direta com a identidade psicológica e sexual do indivíduo. ${ }^{1}$

De acordo com a Organização Mundial da Saúde (OMS), a adolescência pode ser definida como o período da vida situado entre 10 e 19 anos. $^{2}$ Trata-se de uma etapa da vida compreendida entre a infância e a fase adulta, marcada por um complexo processo de crescimento e desenvolvimento biopsicossocial, sendo influenciada por fatores socioculturais, familiares e pessoais.

Diversos aspectos estão envolvidos no aumento de casos de gravidez na adolescência, tais como: influência dos meios de comunicação e da mídia, redução de tabus e inibições sexuais, falta de diálogo e desestruturação familiar, distanciamento entre os conteúdos ministrados em sala de aula e a realidade, menarca precoce, autoafirmação e a gravidez como ritual de passagem da adolescência para a idade adulta. ${ }^{3}$

Aliados a esses fatores, pode-se acrescentar ainda a tendência de queda da idade média da menarca e da iniciação sexual, a falta de informação sobre métodos contraceptivos e a dificuldade de acesso, desconhecimento da fisiologia reprodutiva, como a capacidade de identificar o período fértil, nível de escolaridade e socioeconômico baixo e deficiência de programas de assistência ao adolescente. ${ }^{4,5}$

A gravidez na adolescência é apontada como um problema social. Entretanto, parir antes dos 19 anos, décadas atrás, não se constituía em um problema de saúde pública. As modificações no padrão de fecundidade da população feminina brasileira, as redefinições do papel social da mulher, gerando novas expectativas para as adolescentes, no que diz respeito à escolarização e profissionalização, e o fato de a maioria desses nascimentos acontecerem fora de uma relação conjugal despertam a atenção para o evento. ${ }^{6}$
Os altos índices de gravidez e doenças sexualmente transmissíveis (DSTs) na adolescência denunciam a frequência com que a atividade sexual desprotegida ocorre nessa faixa etária e alertam para a necessidade de uma política de prevenção séria e compromissada. ${ }^{\top}$ Com relação à prevenção, a orientação anticonceptiva consiste em um trabalho educativo que vai além do fornecimento de informações e conhecimentos sobre saúde reprodutiva. $^{7}$

Considerando o desenvolvimento tecnológico relativo ao campo da contracepção e os avanços no âmbito da saúde sexual e reprodutiva, disponibilizar informações e meios no que diz respeito aos métodos contraceptivos existentes é uma das melhores formas de aderir a um programa de prevenção. 0 fato de oferecer opções de escolha desses métodos aos adolescentes gera segurança e, provavelmente, melhor utilização dos mesmos, resultando em uma vida sexual livre de riscos e satisfatória. ${ }^{8}$

Dentre os obstáculos existentes para o uso consistente dos métodos contraceptivos, incluem-se as pressões sociais e os papéis de gênero. Entre eles, destacam-se: a objeção de seu uso pelo parceiro, "o pensar que não engravidaria", ou por "não esperar ter relações naquele momento". ${ }^{5}$

Sabe-se que, na maioria das vezes, o comportamento contraceptivo é sempre posterior ao início do relacionamento sexual. Alega-se que a responsabilidade com relação à vida reprodutiva é atribuição exclusiva da mulher e se deve à imprevisibilidade das relações à não utilização dos contraceptivos. ${ }^{4}$

Diante desse contexto, o presente estudo descreve as práticas em relação ao uso dos métodos contraceptivos de adolescentes das escolas agrícolas da Universidade Federal do Piauí, buscando a associação entre os dados sociodemográficos e conhecimentos sobre os métodos contraceptivos, com o seu respectivo uso.

\section{METODOLOGIA}

Para o desenvolvimento desta pesquisa realizou-se um estudo seccional nas três escolas agrícolas vinculadas à Universidade Federal do Piauí, situadas nas cidades de Teresina, Floriano e Bom Jesus.

A população do estudo foi composta por adolescentes na faixa etária de 14 a 19 anos, regularmente matriculados nas referidas escolas, e foi constituída de 652 alunos (censo), dos quais 185 são da escola de Teresina, 305 de Floriano e 162 de Bom Jesus. Os adolescentes foram convidados a participar da pesquisa e informados sobre o seu tema e objetivos. Utilizaramse como critérios de inclusão: concordar em participar do estudo; estar na faixa etária de 14 a 19 anos de idade e devidamente matriculado em uma das escolas agrícolas da UFPI. Ressalta-se que todos os adolescentes na faixa etária de 14 a 19 anos participaram da pesquisa.

A coleta de dados se deu após a autorização das escolas onde o estudo foi desenvolvido e do Comitê de Ética e Pesquisa 
da Universidade Federal do Piauí, conforme parecer 54/08 respeitando os princípios da Resolução 196/96 do Conselho Nacional de Saúde. Os dados foram coletados por meio de um questionário com perguntas fechadas e algumas mistas, respondido pelo próprio aluno. A coleta dos dados foi realizada pela própria pesquisadora em cada sala de aula, por ocasião dos intervalos entre as aulas, ou no horário da aula, com a permissão do professor. 0 instrumento de coleta de dados incluiu variáveis referentes aos dados sociodemográficos, sexuais e de anticoncepção.

Para se testar a compreensão das questões, realizou-se um pré-teste em uma escola da rede estadual de ensino da cidade de Teresina/PI, com 15 alunos que apresentavam o mesmo perfil dos alunos do estudo, ou seja, adolescentes da faixa etária de 14 a 19 anos.

Os dados foram coletados durante os meses de maio e junho/2008. Utilizou-se o software Epi info versão 3.5/CDC, por meio do qual se calcularam as estatísticas apropriadas aos resultados encontrados, tais como: média e desvio-padrão da idade dos adolescentes do estudo, teste de quiquadrado $\left(\div^{2}\right)$ e teste exato de Fisher para testar diferenças entre as proporções de algumas variáveis (sociodemográficas e conhecimento), com o uso de métodos contraceptivos. Adotou-se o nível de $5 \%$ para o risco de falsa rejeição da hipótese nula com IC (Intervalo de Confiança) de $95 \%$.

\section{RESULTADOS}

\section{Aspectos relacionados à primeira relação sexual da população do estudo}

Tabela 1 - Distribuição de variáveis relacionadas à primeira relação sexual dos adolescentes, segundo o sexo. Teresina/PI, $2009(n=351)$

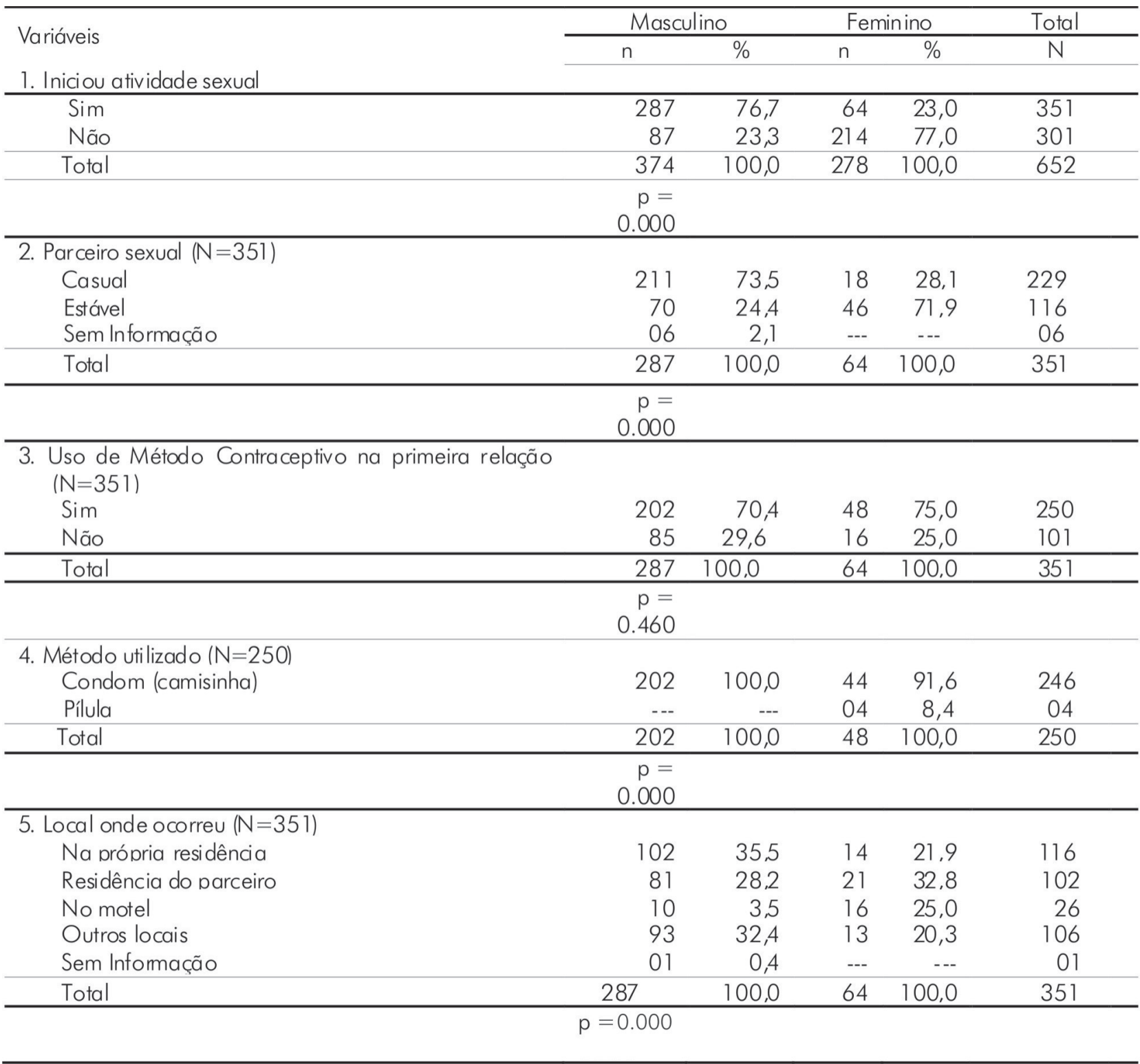


Dos 652 adolescentes estudados, 351 iniciaram a sua atividade sexual, sendo que $287(76,7 \%)$ pertencem ao sexo masculino e $64(23,0 \%)$ ao sexo feminino. Observou-se que a maioria dos adolescentes do sexo masculino teve seu primeiro relacionamento sexual com um parceiro casual $(73,5 \%)$, contrapondo-se aos do sexo feminino que se relacionaram sexualmente com um parceiro de um relacionamento estável $(71,9 \%)$. Na primeira relação sexual, a maioria dos adolescentes de ambos os sexos referiu o uso de algum método contraceptivo, sendo 70,4\% do sexo masculino e $75 \%$ feminino. Com relação ao método utilizado, o condom foi o preferido tanto pelo sexo masculino (100\%) como feminino (91,6\%). 0 local de escolha para terem sua primeira relação sexual foi a própria residência para a grande parte dos adolescentes do sexo masculino (35,5\%), enquanto, para as adolescentes, foi na residência do parceiro (32,8\%) (Tabela 1).

\section{Conhecimento sobre os métodos contraceptivos e fontes de informações}

Tabela 2 - Métodos contraceptivos conhecidos pelos adolescentes do estudo, segundo o sexo e fontes de informação. Teresina/Pl, 2009 ( $\mathrm{n}=652)$

\begin{tabular}{|c|c|c|c|c|}
\hline \multirow{2}{*}{ Métodos Contraceptivos } & \multicolumn{2}{|c|}{ Masculino (374) } & \multicolumn{2}{|c|}{ Feminino (278) } \\
\hline & $\mathrm{n}$ & $\%$ & $\mathrm{n}$ & $\%$ \\
\hline Condom (camisinha) & 360 & 96.3 & 258 & 92.8 \\
\hline Pílula & 313 & 83,7 & 263 & 94,6 \\
\hline Camisinha feminina & 244 & 65,2 & 213 & 76,6 \\
\hline Tabela & 164 & 43,8 & 198 & 71,2 \\
\hline DIU & 163 & 43,6 & 170 & 61,1 \\
\hline Diafraama & 160 & 42,8 & 143 & 51,4 \\
\hline Coito interrompido & 90 & 24,0 & 77 & 27,8 \\
\hline \multirow[t]{2}{*}{ Injetável } & 65 & 17,4 & 84 & 30,2 \\
\hline & $\begin{array}{c}p= \\
0.001\end{array}$ & & & \\
\hline Fontes de Informação & $\mathrm{n}$ & $\%$ & $n$ & $\%$ \\
\hline Escola & 325 & 86,9 & 253 & 91,0 \\
\hline Televisão & 244 & 65,2 & 174 & 62,6 \\
\hline Amigos & 173 & 46,3 & 143 & 51,4 \\
\hline Serviços de Saúde & 193 & 51,6 & 122 & 43,9 \\
\hline Família & 194 & 51,9 & 115 & 41,4 \\
\hline Internet & 111 & 29.7 & 78 & 28,0 \\
\hline Rádio & 54 & 14,4 & 37 & 13,3 \\
\hline \multicolumn{5}{|l|}{ Resposta múltipla } \\
\hline & $\begin{array}{c}p= \\
0.378\end{array}$ & & & \\
\hline
\end{tabular}

Observa-se, na Tabela 2, que, dos métodos contraceptivos mais conhecidos pelos adolescentes do estudo, o condom masculino foi apontado pela expressiva maioria do sexo masculino (96,3\%), seguido da pílula $(83,7 \%)$ e da camisinha feminina $(65,2 \%)$. Com relação ao sexo feminino, os métodos mais conhecidos foram a pílula $(94,6 \%)$ e o condom $(92,8 \%)$, seguido da camisinha feminina $(76,6 \%)$. Os métodos menos conhecidos pela população do estudo foram o coito interrompido e o injetável. No tocante às fontes de informação, as mais citadas pelo sexo masculino foram: a escola (86,9\%), seguida da televisão (65,2\%), família $(51,9 \%)$ e serviços de saúde $(51,6 \%)$. Para o sexo feminino, as informações também provêm da escola ( $91 \%)$, televisão (62,6\%), amigas (51,4\%) e serviço de saúde (43,9\%). 0 rádio e a internet foram os menos referidos. 
Tabela 3 - Associação do uso de contraceptivos com os dados sociodemográficos dos adolescentes. Teresina/Pl, 2009.

\begin{tabular}{|c|c|c|c|c|c|c|}
\hline \multirow{3}{*}{ Variáveis } & \multicolumn{4}{|c|}{ Uso de contraceptivo } & \multirow{2}{*}{\multicolumn{2}{|c|}{ Total }} \\
\hline & \multicolumn{2}{|c|}{ Sim } & \multicolumn{2}{|c|}{ Não } & & \\
\hline & $\mathrm{N}^{\circ}$ & $\%$ & $\mathrm{~N}^{\circ}$ & $\%$ & $\mathrm{~N}$ & $\%$ \\
\hline \multicolumn{7}{|l|}{ Faixa etária (anos) } \\
\hline $14-16$ & 99 & 36,7 & 07 & 43,7 & 106 & 37,0 \\
\hline $17-19$ & 171 & 63,3 & 09 & 56,3 & 180 & 63,0 \\
\hline Total & 270 & 100,0 & 16 & 100,0 & 286 & 100,0 \\
\hline \multicolumn{7}{|c|}{$p=0,569$} \\
\hline \multicolumn{7}{|l|}{ Sexo } \\
\hline Feminino & 50 & 18,5 & 01 & 6,3 & 51 & 17,8 \\
\hline Masculino & 220 & 81,5 & 15 & 93,7 & 235 & 82,2 \\
\hline Total & 270 & 100,0 & 16 & 100,0 & 286 & 100,0 \\
\hline \multicolumn{7}{|c|}{$p=0,213$} \\
\hline \multicolumn{7}{|l|}{ Escolaridade } \\
\hline Médio & 211 & 78,1 & 14 & 43,7 & 225 & 74,5 \\
\hline Técnico & 59 & 21,9 & 18 & 56,3 & 77 & 25,5 \\
\hline Total & 270 & 100,0 & 32 & 100,0 & 302 & 100,0 \\
\hline \multicolumn{7}{|c|}{$p<0,0001$} \\
\hline \multicolumn{7}{|l|}{ Procedência } \\
\hline Interior & 171 & 63,3 & 09 & 56,3 & 180 & 63,0 \\
\hline Capital & 99 & 36,7 & 07 & 43,7 & 106 & 37,0 \\
\hline Total & 270 & 100,0 & 16 & 100,0 & 286 & 100,0 \\
\hline \multicolumn{7}{|c|}{$p=0,569$} \\
\hline
\end{tabular}

Entre os usuários de contraceptivos, identificou-se proporção mais elevada de adolescentes com nível médio, quando comparados aos não usuários $(p<0,0001)$. Ressaltamos ainda que não foi possível identificar diferenças significativas entre usuários ou não de contraceptivos em relação às variáveis faixa etária $(p=0,569)$, sexo $(0,213)$ e procedência $(0,0569)$ (Tabela 3).

Tabela 4 - Associação do conhecimento e uso dos métodos contraceptivos pela população do estudo. Teresina/PI, 2009.

\begin{tabular}{|c|c|c|c|c|c|c|}
\hline \multirow{3}{*}{ Conhecimento } & \multicolumn{4}{|c|}{ Uso de contraceptivo } & \multirow{2}{*}{\multicolumn{2}{|c|}{ Total }} \\
\hline & \multicolumn{2}{|c|}{ Sim } & \multicolumn{2}{|c|}{ Não } & & \\
\hline & $\mathrm{N}^{\circ}$ & $\%$ & $N^{\circ}$ & $\%$ & $\mathrm{~N}$ & $\%$ \\
\hline \multicolumn{7}{|l|}{ Camisinha } \\
\hline Conhece & 263 & 97,8 & 42 & 95,4 & 305 & 97,4 \\
\hline Não conhece & 06 & 2,2 & 02 & 4,6 & 08 & 2,6 \\
\hline Total & 269 & 100,0 & 44 & 100,0 & 313 & 100,0 \\
\hline \multicolumn{7}{|c|}{ Fisher: $p=0,313$} \\
\hline \multicolumn{7}{|l|}{ Pílul a $\left.{ }^{*}\right)$} \\
\hline Conhece & 52 & 19,8 & 06 & 75,0 & 58 & 21,5 \\
\hline Não conhece & 210 & 80,2 & 02 & 25,0 & 212 & 78,5 \\
\hline Total & 262 & 100,0 & 08 & 100,0 & 270 & 100,0 \\
\hline \multicolumn{7}{|c|}{ Fisher: $p<0,002$} \\
\hline \multicolumn{7}{|l|}{ Tabela $\left(^{*}\right)$} \\
\hline Conhece & 10 & 3,8 & - & - & 10 & 3,7 \\
\hline Não conhece & 252 & 96,2 & 08 & 100,0 & 260 & 96,3 \\
\hline Total & 262 & 100,0 & 08 & 100,0 & 270 & 100,0 \\
\hline
\end{tabular}

$\left.{ }^{*}\right)$ Anál ise realizada apenas com o sexo feminino.

Ao buscar-se a associação do conhecimento com o uso dos métodos contraceptivos, observaram-se proporções mais elevadas com relação ao conhecimento e uso da pílula $(p=0,002)$. Não foi possível identificar diferenças significativas entre o conhecimento e uso da camisinha $(p=0,313)$ e da tabela $(p=0,736)$. 0 fato de conhecer a camisinha e utilizá-la não apresentou associação estatística significativa $(p=0,313)$ (Tabela 4$)$. 
Métodos Contraceptivos: A prática dos adolescentes Mendonça RCM, Araújo TME

\section{DISCUSSÃO}

Atualmente, em virtude da precocidade da menarca e da grande oportunidade para manter relações sexuais, devido ao estilo de vida atual e aos estímulos do meio em que se vive, cada vez mais, a iniciação sexual tem ocorrido de uma forma mais precoce. Como consequência, a adolescente está sempre se deparando com situações de risco. ${ }^{8}$

No contexto da iniciação sexual, observa-se neste estudo que mais da metade dos adolescentes do sexo masculino (76,7\%) já iniciaram a atividade sexual, enquanto, na população feminina, ocorreu em menor percentual, pois apenas $23,3 \%$ relataram já ter iniciado sua atividade sexual. A iniciação sexual para as adolescentes foi mais frequente, por volta dos 15 anos (37,5\%), e a idade mediana da sexarca foi de 15,5 anos de idade. Esse achado também está em concordância com outros estudos. $^{8}$

No que se refere aos adolescentes, o maior índice de iniciação sexual ocorreu em torno dos 14 a 15 anos de idade. Percebe-se, portanto, que os adolescentes são estimulados mais precocemente para iniciação sexual. Amaral ${ }^{9}$, em seu estudo sobre a iniciação sexual das adolescentes, realizado em 2006, faz diversas comparações sobre o que representa essa iniciação para ambos os sexos; nesse estudo, ela comenta que os meninos são mais desinibidos e não se preocupam com as consequências, como as doenças sexualmente transmissiveis e a gravidez. Eles dão prioridade ao prazer, não se preocupando em que pode repercutir seu comportamento.

As mulheres geralmente se envolvem mais com as consequências dos seus atos no campo da sexualidade e contracepção, enquanto os homens vivenciam sua sexualidade de uma forma despreocupada e não demonstram preocupação com a contracepção. Essa realidade está associada às desigualdades de gênero presentes em nossa sociedade, pois com relação aos direitos e deveres de homens e mulheres, se atribui à mulher a responsabilidade quanto às práticas de concepção e contracepção e exige do homem uma demonstração de virilidade ${ }^{(10,11)}$.

Diante dessas considerações, faz-se necessário que se tenha conhecimento sobre a idade mais frequente de iniciação sexual dos adolescentes, para que se possam elaborar ações de promoção da saúde sexual e reprodutiva antes de um relacionamento sexual, com a intenção de criar atitudes que reduzam os riscos do sexo desprotegido e promovam um início da vida sexual mais saudável e seguro.

Outro aspecto importante a considerar no início da atividade sexual dos adolescentes deste estudo é com relação ao parceiro sexual. A maioria dos adolescentes iniciou sua atividade sexual com uma parceira casual $(73,5 \%)$, enquanto que, a maioria das adolescentes iniciou sua atividade sexual com um parceiro estável (71,9\%). Essa diferenciação quanto ao tipo de parceiro sexual, é muito bem explicada por Pantoja ${ }^{12}$, o qual relata que, para as adolescentes, sua primeira vez deve ser compartilhada com pessoas de um relacionamento mais duradouro, que envolva também relações afetivas (namoro). Para os adolescentes, a primeira relação é mais para consolidar sua masculinidade, ela tem um caráter aventureiro, geralmente não há vínculo afetivo.

Quanto ao uso de métodos contraceptivos, a expressiva maioria fez uso na primeira relação sexual, com um percentual de $70,4 \%$ e $75 \%$ para o sexo masculino e feminino, respectivamente. 0 método majoritariamente escolhido foi 0 condom (camisinha), com 100\% apontado pelo sexo masculino e 91,6\% pelo sexo feminino. 0 maior uso do preservativo está relacionado principalmente a dois fatores: ao advento da AIDS e ao sucesso de suas campanhas de prevenção, pois essa geração mais nova já nasceu sob o impacto da epidemia, tornando-se mais fácil a adoção do uso do preservativo. Ainda sobre o uso de algum método contraceptivo na iniciação sexual, diversos estudos, 6,13,14 também corroboram esses resultados.

0 uso de condom é frequente na primeira relação sexual, entretanto apresenta descontinuidade e negligência, pois a contracepção é cercada de descuidos, erros e esquecimentos. ${ }^{15}$ Em contrapartida, o fato de usar o preservativo na iniciação sexual aumenta a probabilidade de uso na última relação. Isto é de extrema importância, pois a continuidade dessa prática no intercurso da vida sexual nos leva a reforçar a necessidade de uma orientação contínua para que os adolescentes tenham uma vida sexual saudável e livre de riscos. 0 aprendizado e domínio da contracepção na adolescência é um processo gradual como a iniciação sexual. Os adolescentes estão mais atentos às primeiras relações sexuais do que à continuidade dos intercursos sexuais. ${ }^{16}$

Ao se questionar a população do estudo quanto ao local onde ocorreu a primeira relação sexual, 35,5\% dos adolescentes responderam que foi em sua própria residência, enquanto, para as adolescentes, o local do evento se deu na residência do parceiro (32,8\%). Geralmente a primeira relação sexual não é planejada por parte dos adolescentes, o que se pode constatar pelo local onde ocorreu, ou seja, dentro da casa e/ou na residência do parceiro, sugerindo que foi improvisado. ${ }^{17}$

Com relação ao conhecimento dos adolescentes sobre os métodos contraceptivos, os resultados mostraram que eles conhecem diversos métodos. e os mais citados foram o condom $(96,3 \%)$, seguido da pílula $(83,7 \%)$. Com relação às adolescentes, ocorreu o contrário, pois a pílula foi mais citada, com $94,6 \%$ e o condom ficou em segundo lugar, com $92,8 \%$. A camisinha feminina foi o terceiro método mais referido por ambos os sexos. Nesse estudo, $100 \%$ dos adolescentes tiveram o conhecimento classificado como adequado, uma vez que pelo menos um método contraceptivo era conhecido por todos os investigados.

Diversas pesquisas estão em concordância quando citam o condom e a pílula como métodos mais conhecidos pela grande maioria dos adolescentes. ${ }^{5}$ Observam-se neste estudo que os 
métodos mais conhecidos pelos adolescentes são também os que eles mais utilizam. A maior frequência do uso se deve provavelmente à distribuição gratuita desses métodos pelo sistema de saúde e à própria facilidade encontrada para a sua utilização.

É fato que existe uma grande difusão de informações sobre os métodos contraceptivos por meio das escolas, serviços de saúde, mídia e a própria família. Entretanto, citar os métodos não significa necessariamente conhecê-los, ou seja, ter adquirido informações suficientes sobre as suas vantagens, desvantagens, formas de acesso e modo de usar. Estes aspectos, sem dúvida, ajudariam muito os adolescentes a fazerem opção por algum tipo de método.

Com relação ao fato de ter conhecimento sobre os métodos contraceptivos e não utilizá-los, o estudo 14 "sobre a experiência sexual dos jovens" relata que, em uma pesquisa realizada em São Paulo, no ano de 2004, $87 \%$ dos jovens declararam conhecer os métodos contraceptivos, entretanto, $70 \%$ tiveram a primeira relação sexual sem nenhuma proteção.

Quanto às fontes de informações apontadas pelos adolescentes deste estudo, a escola foi citada pela maioria, totalizando $86,9 \%$ e $91 \%$, para os adolescentes do sexo masculino e feminino, respectivamente. A televisão ocupou 0 segundo lugar dentre as fontes de maior informação, tanto para o sexo masculino $(65,2 \%)$ quanto para o feminino $(62,6 \%)$. A família foi referida pelos adolescentes, com um percentual de $51,9 \%$, enquanto as adolescentes apontaram os amigos como a terceira fonte de informação $(51,4 \%)$.

Não obstante os serviços de saúde tenham sido citados como a quarta fonte de informação, para os adolescentes de ambos os sexos, o percentual atribuído foi relativamente alto, cabendo destacar que esses serviços, mais especificamente por meio da Estratégia de Saúde da Família, começam, ainda que acanhadamente, na nossa realidade, a utilizar o espaço da escola e em parceria com ela levar este tipo de informação aos jovens.

A importância em se colocar um profissional de enfermagem nas escolas baseia-se na possibilidade de elaboração de oficinas sobre saúde sexual e reprodutiva dos adolescentes, no sentido de capacitar os professores, para que abordem o tema em sala de aula, trabalhando os aspectos relacionados à sexualidade e gravidez na adolescência, dando um enfoque na questão da prevenção.

No tocante à escola, é inegável o fato de que ela se constitui em uma das mais importantes fontes de informação. Como promotora de conhecimentos, possui uma função fundamental na educação sexual dos adolescentes. Ressalta-se que, após a obrigatoriedade da abordagem por todos os professores do tema sexualidade, deve ser aproveitada a oportunidade para discutir não somente questões relativas aos comportamentos de risco, mas também aspectos da afetividade, envolvimento, prazer, ou seja, tudo o que está inserido no contexto da sexualidade, que envolva não somente os aspectos biológicos, mas também emocionais, sociais e culturais. Diversos estudos destacam a importância da escola nas ações educativas para ampliar o conhecimento dos adolescentes sobre sexualidade e métodos contraceptivos. ${ }^{18}$

Nos achados desse estudo, percebe-se que, apesar de a internet e do rádio terem sido pouco apontados como fontes de informação, a televisão teve um destaque importante na difusão de informações para os adolescentes de ambos os sexos. Os meios de comunicação, ao transmitirem informações para os adolescentes, se constituem em uma fonte de mais amplo acesso, o que pode ser explicado pelo seu grande poder de difusão. Em alguns estudos, a televisão aparece em primeiro lugar. ${ }^{5}$

Em discordância com este estudo, temos a pesquisa na qual a família ocupa o primeiro lugar como fonte de informações para o adolescente. Neste estudo, também foi possível constatar um fato muito importante no que diz respeito ao papel dos adolescentes como agentes multiplicadores de informações sobre os métodos contraceptivos, pois segundo os resultados, os amigos se constituem em uma fonte de informação importante para as adolescentes $(51,4 \%) .{ }^{11}$

Quanto ao critério de escolha dos métodos contraceptivos pela população do estudo, 68,6\% dos adolescentes e 79,7\% das adolescentes preferem escolher um método que previna tanto a gravidez quanto as doenças sexualmente transmissíveis. Esse resultado nos confirma que grande parte dos adolescentes desse estudo está atenta à questão da prevenção da gravidez e das doenças sexualmente transmissíveis. A segunda opção de escolha foi sobre um método contraceptivo que fosse de fácil acesso.

Nas análises bivariadas, verificou-se significância estatística com relação à variável escolaridade compatível com nível médio e 0 uso dos métodos contraceptivos $(p=0,000)$ e entre conhecimento e uso da pílula $(p=0,002)$. Diante desses resultados, pode-se dizer que os adolescentes conhecem diversos métodos contraceptivos, entretanto existe uma predominância do uso da pílula. Provavelmente, essa opção se deve ao fato de a pílula ser facilmente adquirida nas Unidades de Saúde, e ter relativa facilidade de uso.

\section{CONSIDERAÇÕES FINAIS}

0 estudo sobre a prática dos adolescentes das escolas agrícolas vinculadas à Universidade Federal do Piauí, relacionada aos métodos contraceptivos, nos conduz à reflexão de que a vida sexual dos adolescentes é uma realidade incontestável, e que a iniciação sexual está cada vez mais precoce, o que torna imprescindível proporcionar a esse grupo uma orientação sexual, envolvendo programas educativos que abranjam o contexto sociocultural, educacional, familiar e emocional, nos quais os adolescentes estão inseridos, enfocando a questão dos métodos contraceptivos, uma vez que esse grupo 
necessita de informações mais concretas e sem subterfúgios acerca desse assunto.

Pode-se concluir que, na sua maioria, a população desse estudo pertencia à faixa etária de 14 a 16 anos, era do sexo masculino e tinha, em média, nove anos de estudo. Iniciaram sua atividade sexual com um parceiro casual, sendo o local do acontecimento a sua própria residência ou a residência do parceiro, e a maioria utilizou um método contraceptivo.

0 método contraceptivo mais conhecido e mais utilizado foi o condom masculino e a pílula. Os demais métodos foram citados, entretanto são muito pouco utilizados. Esse fato vem reforçar a necessidade de uma maior divulgação sobre as opções contraceptivas, bem como a disponibilização desses métodos nos serviços de saúde.

Os resultados apontaram também que o principal motivo para a não utilização de um método contraceptivo pelos adolescentes foi "não ter pensado nisso na hora", seguido de achar que não ficaria grávida ou que não engravidaria a parceira.

Com relação às fontes de informação sobre os métodos contraceptivos, a escola se constituiu no principal veículo, mas vale ressaltar que, além dos educadores, os pais e profissionais de saúde são responsáveis pela construção de conhecimentos adequados, que envolvam a sexualidade na adolescência, sobretudo como se prevenir de uma gravidez e/ou uma doença sexualmente transmissível. É importante, então, que eles se disponham a dialogar de uma forma aberta e franca com esses adolescentes, ressaltando a importância da qualidade e da responsabilidade nos relacionamentos afetivos. Além disso, é fundamental que essa educação seja realizada de uma forma integrada (escola, família e Unidades de Saúde), para que tenham mais respaldo para transformar os conhecimentos em atitudes concretas.

Para os adolescentes deste estudo, o principal critério para a escolha de um método contraceptivo foi o que prevenisse tanto a gravidez quanto as doenças sexualmente transmissíveis, esse fato vem confirmar o quanto as campanhas divulgadas na mídia e nos postos de saúde têm conseguido atingir essa população no sentido da conscientização de adoção de medidas preventivas.

Vale salientar, também, que a saúde sexual do adolescente precisa ser discutida no contexto sociopolítico, pois existem poucos programas destinados a essa faixa etária da população, haja vista que a abrangência maior em termos de programas se destina à criança, mulher e idoso, ficando assim o adolescente enquadrado muitas vezes nos programas destinados à criança.

Nesse sentido, é necessário que o governo faça exercer os direitos sexuais e reprodutivos desses adolescentes, fazendose respeitar os princípios de ética, confidencialidade e confiabilidade, para que eles se sintam fortalecidos, amparados e confiantes e possam assim discutir seus problemas e dúvidas relativos à sua sexualidade sem medos e culpas, podendo ter uma vida sexual saudável e livre de comportamentos de riscos.

Com base no estudo realizado, exalta-se a importância do profissional de saúde, especialmente o enfermeiro, nas escolas, planejando e executando trabalhos educativos com destaque à saúde sexual e reprodutiva, por meio de oficinas, com o intuito de formar agentes multiplicadores de saúde, envolvendo o corpo docente, os discentes, pais e lideranças da comunidade. A construção de práticas educativas na escola dá a oportunidade ao adolescente de questionar, se envolver e participar, trabalhando suas próprias dúvidas, permitindo questionamentos e amenizando, portanto, suas angústias, seus tabus e mitos. Isso possibilitará um desenvolvimento mais natural de sua sexualidade.

É importante ressaltar que o profissional de enfermagem, como membro da equipe de saúde, tem um papel significativo na saúde sexual e reprodutiva dos adolescentes. Entretanto, faz-se necessário que esses profissionais sejam preparados para assumir tal função, pois, muitas vezes, sua formação, crenças e valores não estão condizentes com a realidade atual, apresentando dificuldade em acolher e orientar estes adolescentes que os procuram. Sugere-se também que 0 enfermeiro seja inserido nos Conselhos Locais de Saúde, para poder discutir de forma mais abrangente os problemas pertinentes a essa população e fazer encaminhamentos aos órgãos competentes.

\section{REFERÊNCIAS}

1. Oltra RE. Qué experiencias, actitudes y comportamientos tienen los adolescentes españoles ante la contracepción? Revista Cultura de los Cuidados 2003; 2(14): 59-70.

2. World Health Organization. Child and adolescent health development. Geneva: WHO [on line], 2001; [citado em 2007 Nov. 13]; Disponível em http://www.who.int/child-adolescent-health/OVERVIEW/AHD/adhover.htm.

3. Heidemann M. Adolescência e Saúde: uma visão preventiva: para profissionais de saúde e educação. Petrópolis (RJ): Vozes; 2006.

4. Cabral CS. Contracepção e Gravidez na adolescência na perspectiva de jovens pais de uma comunidade favelada no Rio de Janeiro. Cad. Saúde Pública. 2003; 19(52): 83-92.

5. Belo MAV, Silva JLP. Conhecimento, atitude e prática sobre métodos anticoncepcionais entre adolescentes gestantes. Rev. Saúde Pública. 2004; 38(4): 479-87.

6. Brandão ER, Heilborn ML. Sexualidade e gravidez na adolescência entre jovens de camadas médias do Rio de Janeiro, Brasil. Cad. Saúde Pública. 2006; 22(70): 1421-30.

7. Leal MM, Amado CR. Anticoncepção na adolescência. In: Gejer D. Sexualidade e saúde reprodutiva na adolescência. São Paulo: Atheneu; 2001.

8. Berlofi LM. Prevenção da reincidência de gravidez em adolescentes: efeitos de um Programa de Planejamento Familiar. Acta Paul. Enferm. 2006; 19(2): 196-200. 
9. Amaral MA, Fonseca RMGS. Entre o desejo e o medo: as representações sociais das adolescentes acerca da iniciação sexual. Rev. Esc. Enferm. USP. 2006; 40(4): 469-76.

10. Arcanjo CM, Oliveira MIV, Bezerra MGA. Gravidez em adolescentes de uma Unidade Municipal de Saúde em Fortaleza - Ceará. Esc Anna Nery Rev Enferm 2007 set.; 11(3): 445-51.

11. Rangel DLO, Queiroz ABA. A Representação social das adolescentes sobre a gravidez nesta etapa de vida. Esc Anna Nery Rev Enferm 2008 dez.; 12(4): 780-88.

12. Pantoja ALN. "Ser alguém na vida": uma análise sócio-antropológica da gravidez/maternidade na adolescência, em Belém do Pará, Brasil. Cad. Saúde Pública. 2003; 19(2): 335-43.

13. Almeida MCC, Aquino EML, Gaffikin L, Magnan R. Uso de contracepção por adolescentes de escolas públicas na Bahia. Rev. Saúde Pública. 2003; 37(5): 566-75.
14. Villela WV, Doreto DT. Sobre a experiência sexual dos jovens. Cad. Saúde Pública. 2006; 22(11): 2467-72.

15. Teixeira AMFB, Knauth DR. Adolescentes e uso de preservativos: as escolhas dos jovens de três capitais brasileiras na iniciação e na última relação sexual. Cad. Saúde Pública. 2006; 22(7): 1385-96.

16. Borges ALV, Schor N. Início da vida sexual na adolescência e relações de gênero: um estudo transversal em São Paulo, Brasil, 2002. Cad. Saúde Pública. 2005; 21 (2): 499-507.

17. Bourscheid JL. Sexualidade: Reconstruindo compreensões de forma coletiva, partindo da visão dos adolescentes. [dissertação de mestrado]. Porto Alegre: Pontifícia Universidade Católica do Rio Grande do Sul; 2004.

18. Tornis NHM, Lino AIA. Sexualidade e anticoncepção: o conhecimento do escolar/adolescente. Rev. Eletr. Enf. 2005; 7(3): 344-50

\section{NOTA}

a Parte da dissertação defendida no PPG Mestrado em Enfermagem, em março de 2009. 